\title{
Positionspapier des Verbandes pneumologischer Kliniken und der Deutschen Gesellschaft für Pneumologie und Beatmungsmedizin zur Kodierung der invasiven und nicht-invasiven Beatmung bei intensiv- medizinisch versorgten Patienten
}

\author{
Statement of the Association of Pneumological Clinics and the German Respiratory Society \\ on the Coding of Invasive and Non-Invasive Ventilation in Intensity Care
}

Bibliografie

DOI http://dx.doi.org/

10.1055/s-0033-1344210

Pneumologie 2013; 67: 371-375

(c) Georg Thieme Verlag KG

Stuttgart · New York

ISSN 0934-8387

Korrespondierender Autor

Prof. Dr. med.

Winfried Randerath

Krankenhaus Bethanien gGmbH

Aufderhöher Str. 169

42699 Solingen

randerath@klinik-bethanien.de

\section{Zusammenfassung}

\section{$\nabla$}

Die Beatmung respiratorisch insuffizienter Patienten stellt einen wichtigen Schwerpunkt der intensivmedizinischen Versorgung dar. Je nach klinischer Situation und zugrundeliegendem Krankheitsbild kann sie mittels invasivem oder nicht-invasivem Zugang durchgeführt werden. Abrechnungsgrundlage der Vergütung stellt im System diagnosebezogener Fallgruppen (DRGSystem) der Aufwand und Ressourcenverbrauch für einen Krankheitsfall dar. Dieser richtet sich bei beatmeten Patienten nach der Anzahl der Beatmungsstunden und ist in den deutschen Kodierrichtlinien festgelegt. Mit diesem Positionspapier stellen die Deutsche Gesellschaft für Pneumologie und Beatmungsmedizin und der Verband pneumologischer Kliniken einige Punkte der Kodierrichtlinien klar, die bei invasiv und nicht-invasiv beatmeten Patienten in der intensivmedizinischen Versorgung von Relevanz sind.

Die Betreuung von Beatmungspatienten nimmt in der klinischen Versorgung einen großen Rahmen ein. Dies stellt einen hohen Anspruch an die Qualität der Versorgung, der mit entsprechendem personellen Aufwand (Ärzte, Pflegepersonal) und materiellen Ressourcen verbunden ist [1-2]. Das ist die Basis für die Kodierung der Beatmungsstunden. Diese unterliegen der Überprüfung durch den Medizinischen Dienst der Krankenkassen. Aus Sicht der Deutschen Gesellschaft für Pneumologie und Beatmungsmedizin und des Verbandes pneumologischer Kliniken sind einige Punkte, die in den Kodierrichtlinien definiert und die für die Einschätzung der Beatmung in der intensivmedizinischen Versorgung grundlegend sind, klarzustellen und festzuhalten. Das Papier hat die Kodierung der invasiven und nicht-invasiven Beatmung bei akuten Erkrankungen, Verschlechterungen der chronischen respiratorischen Insuffizienz, in der Langzeitbeatmung und

\section{Abstract \\ $\nabla$}

Mechanical ventilation in patients with respiratory failure represents one of the most important aspects of intensity care. It can be performed invasively and non-invasively depending on the clinical situation and the underlying disease. The expenditure and consumption of resources is the basis of the compensation for each patient case in the German diagnosis related group system. For ventilated patients it is calculated based on the hours of ventilation, according to the standard coding guideline. In this statement, the German Respiratory Society and the Association of Pneumological Clinics aim to clarify some aspects of the coding of invasive and non-invasive ventilation.

dem Weaning von der Beatmung, jeweils ausschließlich in der intensivmedizinischen Versorgung, zum Inhalt. Es beschäftigt sich nicht mit der elektiven Neueinleitung oder Kontrolle einer nicht-invasiven Beatmung bei Patienten in der stabilen Situation einer chronisch respiratorischen Insuffizienz. Die medizinische Indikationsstellung und Differenzialtherapie der invasiven oder nicht-invasiven Beatmung sind in wissenschaftlichen Leitlinien beschrieben [1 - 2] und daher nicht Gegenstand dieses Papiers, das lediglich die Kodierung der Beatmungsstunden der beim einzelnen Patienten durchgeführten invasiven oder nicht-invasiven Beatmung vorstellt.

1.

Die deutschen Kodierrichtlinien [3] definieren die maschinelle Beatmung über die Bewegung von Gasen mittels einer mechanischen Vorrichtung. Neben der invasiven Beatmung ( $\bullet$ Abb.1) 


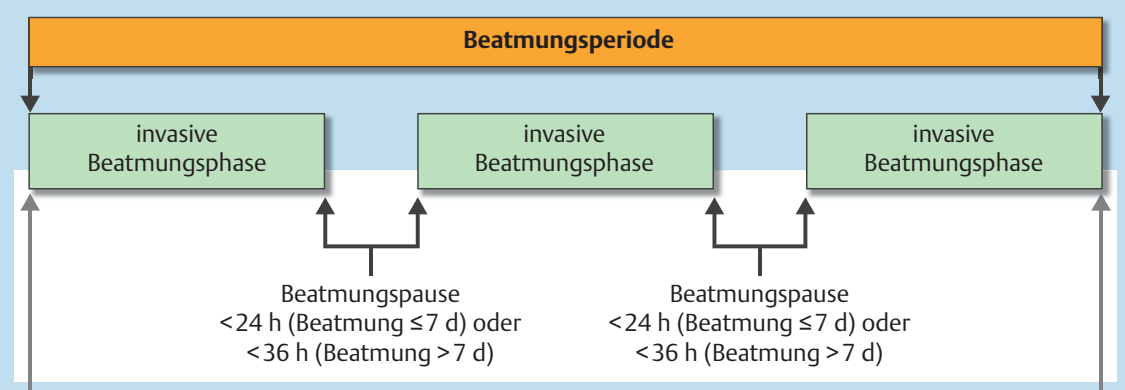

Abb. 1 Invasive Beatmung

Beginn der Beatmung mit

Intubation oder

Tracheotomie oder

Aufnahme eines beatmeten Patienten
Ende der Beatmung durch

Extubation oder

Entwöhnung oder

Entlassung oder

Verlegung von der Intensivstation oder über Intubation oder Tracheotomie kann die maschinelle Beatmung auch über Maskensysteme erfolgen, wenn diese Intubation oder Tracheotomie ersetzen $(\bullet$ Abb. 2) [3].

Unter einer Maskenbeatmung ist eine nicht-invasive Beatmung zu verstehen.

Nicht jede nicht-invasive Beatmung führt zur Kodierung von Beatmungsstunden. Voraussetzungen sind, dass

- der Patient intensivmedizinisch versorgt ist

- die nicht-invasive Beatmung die Intubation oder Tracheotomie ersetzt.

Die Maskenbeatmung kann die invasive Beatmung (nach orotrachealer Intubation oder Tracheotomie) unter Beachtung eventueller Kontraindikationen in allen Situationen ersetzen, in denen die Spontanatmung des Patienten nicht ausreichend ist [4-7]:

akute hypoxische oder hyperkapnische Insuffizienz

(ه Abb. 2, 3).

- akute Verschlechterung bei einem Patienten mit einer chronischen hypoxischen oder hyperkapnischen Insuffizienz

(๑ Abb.4).

- Fortführung der Beatmung im Weaning von zuvor invasiv beatmeten Patienten ( $\bullet$ Abb.5).

Bei verschiedenen Erkrankungen, wie zum Beispiel einer kardialen Dekompensation, einer Pneumonie oder einer Niereninsuffizienz, kann es konsekutiv zur Atmungsinsuffizienz kommen, sodass eine maschinelle Beatmung erforderlich wird. Eine nicht-invasive Beatmung kann hier eine Intubation vermeiden ( Abb. 2,3).

Patienten, bei denen bereits eine chronische respiratorische Insuffizienz vorliegt und die deswegen bereits invasiv (über Tracheostoma) oder nicht-invasiv beatmet werden, können im Rahmen der Grunderkrankung, einer anderen vorbestehenden Erkrankung oder einer neuen Erkrankung eine akute Verschlechterung erfahren. Ein Teil muss dann intensivmedizinisch versorgt und intensiviert oder dauerhaft beatmet werden. Dies kann bei häuslich chronisch nicht-invasiv beatmeten Patienten auch mit einer Intensivierung der nicht-invasiven Beatmung erfolgen, sofern keine Kontraindikationen vorliegen. Auch in dieser Situation ersetzt die jetzt intensivierte nicht-invasive Beatmung die sonst notwendige invasive Beatmung. Es ist eine neue Krankheitssituation aufgetreten, die sich auf die vorher stabile chronische Situation aufgesetzt hat ( $\bullet$ Abb.4).

Im Entwöhnungsprozess von einer maschinellen Beatmung (Weaning) kann eine Extubation mit anschließendem Übergang auf die nicht-invasive Beatmung folgen. Dies ist heute ein Standardverfahren im Entwöhnungsprozess. Die frühzeitige Extuba- tion und anschließende nicht-invasive Beatmung ermöglicht eine Verkürzung der invasiven Beatmungszeit, eine Verringerung der Mortalität und des Intensivaufenthaltes. Die nicht-invasive Beatmung ersetzt also an dieser Stelle eine ansonsten länger durchzuführende invasive Beatmung $[8,9]$ ( $\bullet$ Abb.5).

\section{2.}

Aus den oben dargelegten Aspekten und der Definition der maschinellen Beatmung nach den Kodierrichtlinien ergibt sich, dass eine nicht-invasive Beatmung sowohl in der Akutsituation, bei der akuten Verschlechterung einer chronischen Situation, als auch in der Entwöhnung von der Langzeitbeatmung die invasive Beatmung ersetzen kann [1-3].

3.

Die Dauer der Beatmung wird durch den Beginn und das Ende in den Kodierrichtlinien definiert. Hier legen die Kodierrichtlinien den Beginn klar über die endotracheale Intubation, den Beginn der Maskenbeatmung, die Tracheotomie oder die stationäre Aufnahme eines maschinell beatmeten Patienten fest. Das Ende wird durch die Extubation, die Beendigung der Beatmung nach einer Periode der Entwöhnung, die Entlassung des Patienten oder die Verlegung definiert.

Der Gesamtzeitraum von Beginn der Beatmung bis zu ihrem Ende stellt nach den Richtlinien eine Beatmungsperiode dar ( Abb. 1-6) [3]. Es ist denkbar, dass der Patient mehrere Beatmungsperioden während eines Krankenhausaufenthaltes erfährt. Dies kann zum Beispiel vorliegen, wenn ein Patient nach einer Operation über einen Tag beatmet, dann extubiert wird und nach mehreren Tagen im Rahmen einer Lungenembolie oder einer Aspiration eine erneute respiratorische Insuffizienz erleidet und erneut beatmet werden muss. Beide Perioden wären von Beginn der Beatmung, also Einleitung der Operation, bis zur Extubation (1.Periode) und anschließend von der Reintubation nach mehreren Tagen Normalatmung bis zur definitiven Extubation berechnet (2.Periode) ( $\bullet$ Abb.6).

Innerhalb einer Beatmungsperiode muss dabei der Patient keineswegs durchgehend (also 24 Stunden/Tag) beatmet sein. Vielmehr können Phasen der Beatmung durch Phasen der Spontanatmung zum Zwecke der Entwöhnung unterbrochen werden. Hierzu sind in den Kodierrichtlinien klare Vorgehensweisen mit Zeitkorridoren je nach Dauer der Beatmung definiert. Die Phasen der Beatmungsunterbrechungen zählen in diesem Rahmen vollständig als Beatmungsstunden. Die Beatmungsdauer wird also vollständig für die gesamte Beatmungsperiode, also sämtliche 


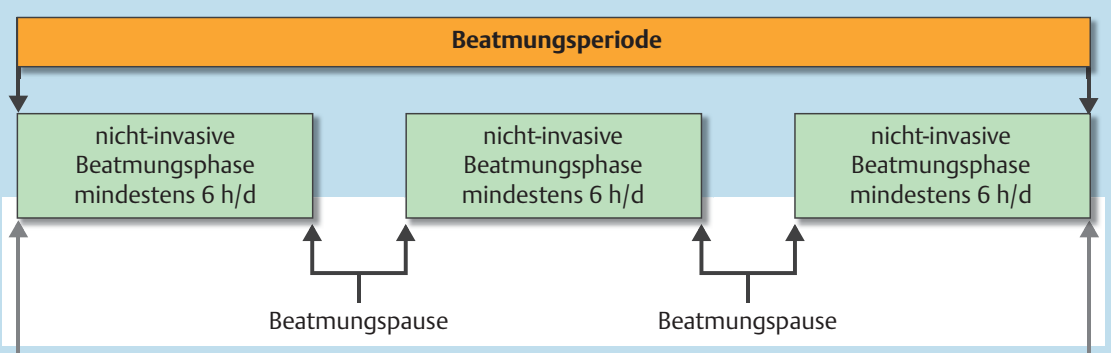

Beginn der Beatmung mit akuter Einleitung nicht-invasiver Beatmung (Maske);

Verhinderung der Intubation;

Voraussetzung intensivmedizinische Betreuung

Ende der Beatmung durch

Entwöhnung oder

Entlassung oder

Verlegung von der Intensivstation oder
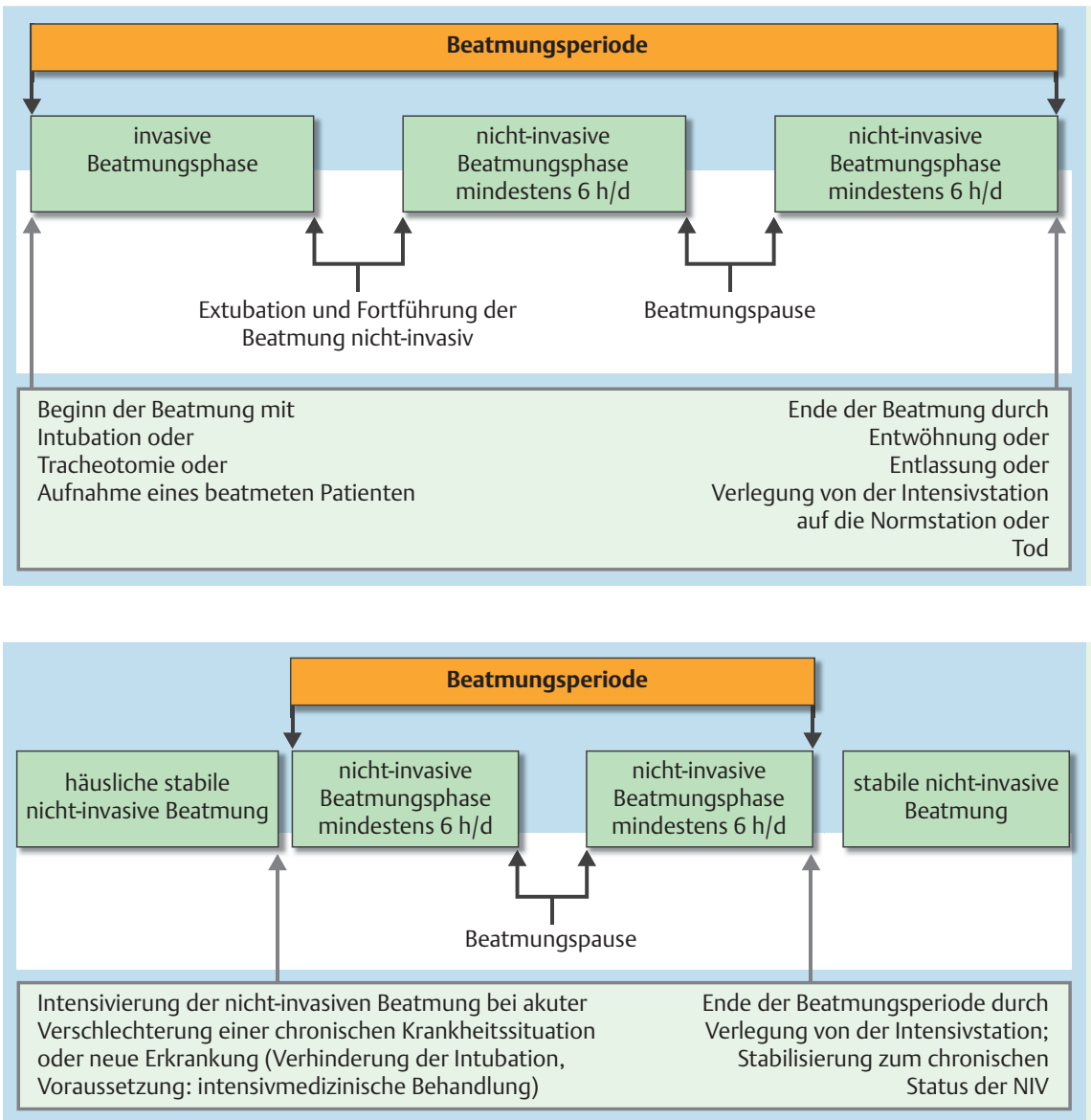

Abb.4 Intensivierung einer vorbestehenden nichtinvasiven Beatmung bei akuter Verschlechterung zur Verhinderung der Intubation.

Abb.3 Invasive und anschließende nicht-invasive Beatmung in der Akuttherapie.

Abb. 2 Nicht-invasive Beatmung als Akutbeatmung zur Verhinderung der Intubation.

Beatmungsphasen und Spontanatmungsphasen, berechnet. Keineswegs kann eine Beatmungsphase als Beatmungsperiode verstanden werden und somit auf tatsächlich beatmeten Stunden kalkuliert werden. Eine Beatmungsperiode ist die Summe aus Beatmungsphasen und Phasen der Spontanatmung ( Abb.1-6) [3].

Diese Kodierung steht in Übereinstimmung mit dem Prinzip des DRG-Systems, das den Aufwand der Therapie zugrunde legt. Die Spontanatmungsphasen innerhalb der Beatmungsperioden stellen jedoch keineswegs einen geringeren Aufwand dar als die Beatmungsphasen, da hier ein gesteigerter Aufwand in Überwachung, Unterstützung und Begleitung des Patienten notwendig ist.

\section{4.}

Es gibt keinen Unterscheid in der Berechnung der Beatmungsperioden, also der Summe von Atmungsphasen und beatmungsfreien Phasen, hinsichtlich der Art der Beatmung. Die nicht-invasive Beatmung wird also in gleicher Weise wie die invasive Beatmung kalkuliert, es gelten die gleichen Bedingungen für Anfang und Ende der Beatmung. Ein Unterschied ergibt sich lediglich für den Zeitpunkt des Endes nach der Entwöhnung (siehe 6.).

5.

Eine nicht-invasive Beatmung kann nach den Kodierrichtlinien nach verschiedenen Methoden erfolgen. Insbesondere werden sowohl assistierte nicht-invasive Beatmung als auch andere Formen Atemunterstützung berücksichtigt [3]. 

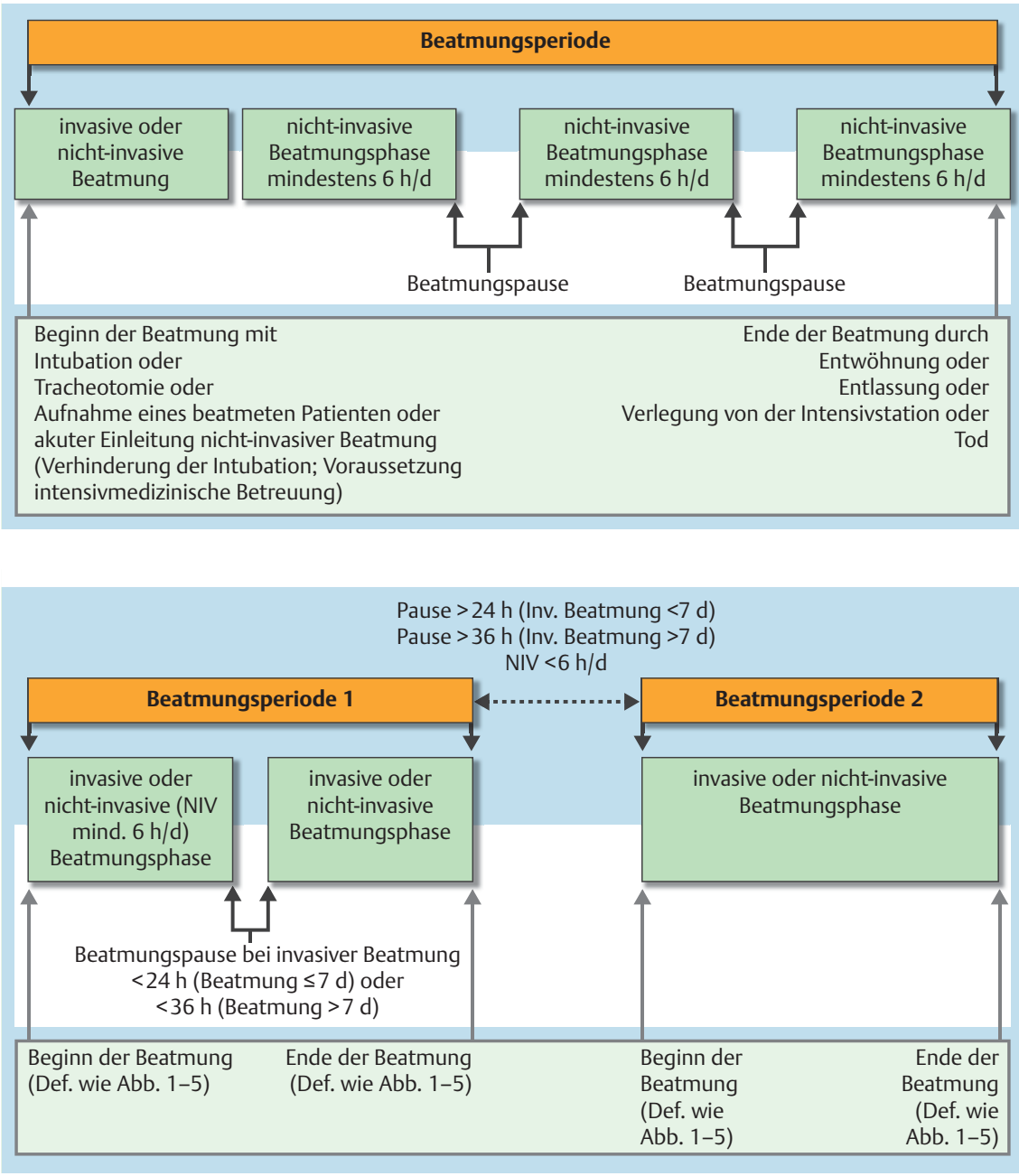

Abb.5 Nicht-invasive Beatmung im Weaning.

Abb. 6 Mehrere unabhängige Beatmungsperioden während eines stationären Aufenthaltes.
6.

Die Kodierrichtlinien unterscheiden sich in invasiver und nichtinvasiver Beatmung im Hinblick auf das Ende der Beatmung. Bei der invasiven Beatmung ist die Entwöhnung des Patienten beendet, wenn eine stabile respiratorische Situation über 24 Stunden bei bis zu 7 Tagen Beatmung oder 36 Stunden bei mehr als 7 Tagen Beatmung vorliegt.

Im speziellen Fall einer Entwöhnung mit intermittierenden Phasen der maschinellen Unterstützung der Atmung durch nicht-invasive Beatmung im Wechsel mit Spontanatmung ist eine Anrechnung auf die Beatmungszeit nur möglich, wenn die Spontanatmung des Patienten insgesamt mindestens 6 Stunden pro Kalendertag nicht-invasiv unterstützt wurde. Die Berechnung der Beatmungsdauer endet in diesem Fall nach der letzten MaskenCPAP-Phase an dem Kalendertag, an dem der Patient zuletzt insgesamt mindestens 6 Stunden durch Masken-CPAP unterstützt wurde ( Abb.5) [3].

\section{Zusammengefasst bleibt festzuhalten:}

1. Bei invasiv beatmeten Patienten ist die gesamte Beatmungszeit von Beginn der Beatmung bis zu ihrem Ende vollständig zu kodieren.

2. Die nicht-invasive Beatmung wird in gleicher Weise kodiert wie die invasive Beatmung. Ein Unterschied ergibt sich lediglich beim Ende der Beatmung. Dies liegt vor, wenn der Patient weniger als 6 Stunden pro 24 Stunden nicht-invasiv beatmet wurde.
3. Bei Patienten mit chronischer hypoxischer oder hyperkapnischer Insuffizienz, die stabil außerklinisch invasiv oder nichtinvasiv beatmet werden, kann es zu einer akuten Verschlechterung kommen, die die vorbestehende respiratorische Insuffizienz verstärkt. Diese akute Verschlechterung ist eine neue Erkrankung. Falls diese zum Aufenthalt auf der Intensivstation führt, sind die Beatmungszeiten vom Beginn der Therapieintensivierung im Krankenhaus bis zu ihrem Ende zu kodieren. Das Ende wird durch die Wiederherstellung einer stabilen Beatmungssituation entsprechend der Ausgangslage definiert.

4. Bei Patienten, die im Rahmen einer chronisch hyperkapnischen Insuffizienz elektiv (also nicht in einer Akutsituation) zur Einleitung oder Kontrolle einer nicht-invasiven Beatmung aufgenommen werden und die nicht-intensivmedizinisch betreut werden, sind keine Beatmungsstunden zu kodieren, auch wenn diese Einstellung auf einer Intensivstation erfolgen sollte.

5. Invasiv beatmete Patienten und kontinuierlich vom Respirator abhängige Patienten unter NIV sind aus Gründen der Überwachung und Betreuung grundsätzlich intensivmedizinisch zu versorgen, da zum Beispiel bei akzidentiellen Dekanülierungen, Geräteausfall oder Kanülenverlegung eine vitale Bedrohung bestünde. Eine nicht-intensivmedizinische Versorgung dieser Patienten würde eine Verschlechterung gegenüber der 1:1-Versorgung zu Hause oder der heimpflegerischen Betreuung in einer Beatmungspflegeeinrichtung darstellen. 


\section{Interessenkonflikt}

$\nabla$

Der federführende Ansprechpartner ist Chefarzt einer pneumologischen Fachklinik, in der auch beatmete Patienten betreut werden.

\section{Literatur}

1 Windisch W, Brambring J, Budweiser $S$ et al. Nichtinvasive und invasive Beatmung als Therapie der chronischen respiratorischen Insuffizienz. S2-Leitlinie herausgegeben von der Deutschen Gesellschaft fur Pneumologie und Beatmungsmedizin e.V. Pneumologie 2010; 64: 207 240

2 Schönhofer B, Kuhlen R, Neumann P et al. Nichtinvasive Beatmung als Therapie der akuten respiratorischen Insuffizienz. Pneumologie 2008; 62: $449-479$

3 Institut für das Entgeltsystem im Krankenhaus (InEK GmbH). Deutsche Kodierrichtlinien Version 2013 (DKR) Lich: Pictura; 2012
4 Demoule A, Girou E, Richard JC et al. Benefits and risks of success or failure of noninvasive ventilation. Intensive Care Med 2006; 32: $1756-1765$

5 Masip J, Roque M, Sanchez B et al. Noninvasive ventilation in acute cardiogenic pulmonary edema: systematic review and meta-analysis. JAMA 2005; 294: $3124-3130$

6 Weng CL, Zhao YT, Liu QH et al. Meta-analysis: Noninvasive ventilation in acute cardiogenic pulmonary edema. Ann Intern Med 2010; 152: $590-600$

7 Ram FS, Picot J, Lightowler J et al. Non-invasive positive pressure ventilation for treatment of respiratory failure due to exacerbations of chronic obstructive pulmonary disease. Cochrane Database Syst Rev 2004: CD004104

8 Ferrer $M$, Sellares J, Valencia $M$ et al. Non-invasive ventilation after extubation in hypercapnic patients with chronic respiratory disorders: randomised controlled trial. Lancet 2009; 374: $1082-1088$

9 Nava S, Gregoretti C, Fanfulla F et al. Noninvasive ventilation to prevent respiratory failure after extubation in high-risk patients. Crit Care Med 2005; 33: $2465-2470$ 